\title{
Running economy - a comprehensive review for passive force generation
}

\author{
Christos Ziliaskoudis', Song-Young Park', Sang-Ho Lee ${ }^{2 * *}$ \\ ${ }^{1}$ School of Health and Kinesiology, University of Nebraska Omaha, Omaha, NE, USA \\ ${ }^{2}$ Department of Taekwondo Mission, Kosin University, Busan, Korea
}

Running economy is considered a major determinant of distance running performance. Enhancing the body's ability for passive force generation could have a positive effect on running economy by minimizing the energy cost required for the propulsion of the body. Thus, the purpose of this comprehensive review was to provide a list of modifiable factors that promote this ability. The interest was focused on lower-limb stiffness, as it is a factor of great influence and at the same time can be modified with training and specific biomechanical adjustments. Al- though it appears that no clear instructions can be provided to athletes and coaches, it should be noted that careful consideration of the runners' anthropometric, physiological, and biomechanical characteristics are necessary for optimal performance results.

Keywords: Running economy, Passive force generation, Lower-limb stiffness

\section{INTRODUCTION}

Running economy has been defined as the steady-state oxygen consumption for a determined, constant, submaximal running speed (Barnes and Kilding, 2015). The physiologically steady state achieved by running at constant speed allows for the amount of consumed adenosin triphosphate to be better reflected (Fletcher et al., 2009). Moreover, running at submaximal speed, which prevents the accumulation of blood lactate, reassures that only aerobic mechanisms contribute to the energy production procedures (Fletcher et al., 2009). Running economy has gained popularity as a long-distance running performance criterion due to its proposed ability to explain performance variations among runners with similar levels of fitness, as evaluated by conventional measures (Conley and Krahenbuhl, 1980). Running economy has been characterized as a complex and multifactorial measure being affected by a plethora of environmental, physiological, and biomechanical factors as well as by their interplay (Barnes and Kilding, 2015). Training interventions optimizing each of the above factors can enhance running economy. The majority of literature has been focused on the promotion of physiological adaptations. To our knowledge, comprehensive reviews investigating the effect of lower-limb stiffness on running economy are in paucity. For this reason, this review will focus on discussing the impact of lower-limb stiffness on the promotion of passive, metabolically nontaxing force generation as a potential mechanism for enhancing running economy. A practical-perspective approach was prioritized as a way to update and critically assess the related literature with the goal of providing applicable training recommendations in response to the increasing interest for further improvements in distance running performance. In the same concept, critique on common training interventions is provided.

\section{MODIFIABLE FACTORS RELATED WITH PASSIVE FORCE GENERATION}

During running, active force generation for body weight support and forward propulsion represents $80 \%$ of the total metabolic cost
*Corresponding author: Sang-Ho Lee (iD https://orcid.org/0000-0001-9935-317X Department of Taekwondo Mission, Kosin University, 194 Wachi-ro, Yeongdo-gu, Busan 49104, Korea

E-mail: stranger03@kosin.ac.kr

Received: July 22, 2019 / Accepted: August 20, 2019
This is an Open Access article distributed under the terms of the Creative Commons Attribution Non-Commercial License (http://creativecommons.org/licenses/by-nc/4.0/) which permits unrestricted non-commercial use, distribution, and reproduction in any medium, provided the original work is properly cited. 
(Arellano and Kram, 2014). Logically, reducing the requirements of active force generation would reduce the total metabolic cost of running and improve running economy. A way for this to be achieved is by enhancing the contribution of mechanisms related with passive force generation. Due to the purpose of the review, focus will be placed on the practically modifiable factors. Under this perspective, lower-limb stiffness is proposed to have a significant effect on the body's ability to store and return elastic energy (Moore, 2016). This is rather important, if we consider that during running $40 \%-50 \%$ of the total energy requirements are being covered by elastic energy (Moore, 2016).

\section{Lower-limb stiffness}

Leg function during running locomotion has been paralleled with the behavior of a spring that is constantly compressed and decompressed (Cavagna et al., 1988). Mainly responsible for the leg-spring compression is the body's mass. Specifically, the eccentric phase of stance represents the compression of the leg-spring, during which mechanical energy is being stored. The concentric phase of stance represents the decompression of the leg-spring, which is accompanied by the release of the stored mechanical energy in the form of elastic energy. Given its elastic properties, the leg-spring, as every conventional spring, tends to resist to any deforming force. The magnitude of this resistance depends on the leg-spring's stiffness. Similarly, at the leg-spring decompression the magnitude of the returned elastic energy is positively related with stiffness (Dalleau et al., 1998).

Kerdok et al. (2002) highlighted the distinction between $\mathrm{k}_{\operatorname{leg}}$ and $k_{\text {vert. }}$ Specifically, $k_{\operatorname{leg}}$ describes the mechanical behavior of the leg's structural components (i.e., muscles, tendons, and ligaments), as it is compressed during the early phase of stance. In contrast, $\mathrm{k}_{\text {vert }}$ describes the runner's global compression, as expressed by the vertical reversal of the center of mass (COM) velocity's direction (Kerdok et al., 2002). However, it has been proposed that $\mathrm{k}_{\text {vert }}$ is not able to detect the different contribution level of each joint to the determination of the whole leg's stiffness (Hamill et al., 2014). For this reason, the concept of joint stiffness $\left(\mathrm{k}_{\text {joint }}\right)$ was introduced, which models the relationship between joint moment and joint angle. For the calculation of $\mathrm{k}_{\text {joint }}$, the vertical load in the case of $\mathrm{k}_{\text {vert }}$ is being substituted by the joint mo-

$\quad$ Striking Pattern:
Forefoot landing
- Increased knee joint stiffness
- Decreased ankle joint stiffness
- Increased muscle activation
requirements
- Rearfoot landing
- Increased ankle joint stiffness
- Decreased knee joint stiffness
- Wide prevalence among runners of
different levels

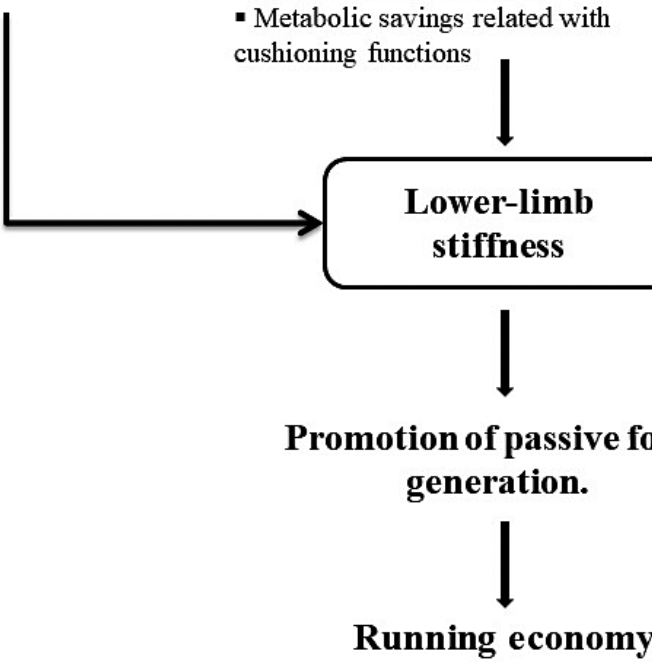

\section{Footwear:}

Barefoot running

- Increased leg stiffness

- Unaltered vertical leg stiffness

- Increased total work production due to greater displacement of the COM (net efficiency)

Shod running

- Disadvantageous in terms of storage

and return of elastic energy

- Lower total work demands

- Metabolic savings related with

cushioning function

\section{Neuromuscular Training Interventions:}

- Musculotendinous tissue modifications favoring stiffness

- Secondary performance variables have been utilized for the establishment of correlations with running economy

Fig. 1. Diagram for the factors affecting running economy. COM, center of mass. 
ment value, and the vertical displacement of the COM is being replaced by the change in the joint angle under the general concept of a torsion spring (Hamill et al., 2009).

Given the established inverse relationship among the various lower-limb stiffness measures and the metabolic cost of running due to enhanced passive force generation as a consequence of the increased return of elastic energy (Dalleau et al., 1998; Spurrs et al., 2003), a list of modifiable factors potentially contributing to enhanced leg stiffness and subsequently improved running economy will follow (Fig. 1).

\section{Striking pattern}

The way a runner's foot comes in contact with the ground, the striking pattern, appears to be related with the behavior of the different lower-limb stiffness measures (Hamill et al., 2014; Laughton et al., 2003; Lieberman et al., 2010; Melcher et al., 2017; Shih et al., 2013). Particularly, a forefoot striking pattern, in which the ball of the foot touches the ground before the heel does (Lieberman et al., 2010), has been related with increased knee joint stiffness and decreased knee range of motion (ROM), and decreased ankle joint stiffness and increased ankle ROM. In the case of a rearfoot striking pattern, the relationships are reversed (Hamill et al., 2014; Laughton et al., 2003; Lieberman et al., 2010; Melcher et al., 2017). Since these measurements of joint stiffness are regulated by the ratio of joint moment to joint angle $(\Delta \mathrm{M} / \Delta \theta)$, one potential mechanistic explanation for these observations could be that in terms of forefoot striking, the increased ankle ROM is responsible for the decreased ankle joint stiffness, while the decreased knee ROM is responsible for the increased knee joint stiffness. Again, in the case of a rearfoot striking pattern the relationship is reversed (Melcher et al., 2017). As mentioned, these interactions describe the effect of the striking pattern on joint stiffness measurements. From a more global perspective, the spring-like behavior of the leg during running is better described by the measure of leg stiffness rather than the individual stiffness of each joint. For this reason, it would be beneficial to examine how the magnitude of global leg stiffness is being affected by the variations of ankle and knee joint stiffness values as they interact with the type of striking pattern. In this regard, the literature appears to be divided (Farley and Morgenroth, 1999; Laughton et al., 2003). Farley and Morgenroth (1999) proposed that leg stiffness is more sensitive to ankle stiffness. This selective sensitivity appears to be explained by the leg's geometry. The length of the foot oriented horizontally creates a longer ground reaction force moment arm which is related with higher moment and angular dis- placements relative to the knee and hip joints. Thus, an absolute increase in ankle stiffness by a rearfoot striking pattern would be more influential on the global leg stiffness. The experimental intervention of this study, however, was a hoping task (Farley and Morgenroth, 1999). This idea of increased leg stiffness dependence on ankle joint stiffness is further supported by the reported correlation between the Achilles tendon moment arm, which coincides with the length of the calcaneal tuberosity, and running economy. Shorter Achilles tendons' moment arms were correlated with improved running economy and were found to explain a significant percentage $(58 \%-81 \%)$ of the total metabolic cost variance (Lacour and Bourdin, 2015). On the other hand, Laughton et al. (2003) proposed the knee as the joint of greater influence on leg stiffness. The simultaneous increase in knee joint stiffness and leg stiffness along with the reduction in ankle joint stiffness observed during the forefoot running experimental condition of the study was used as an argument (Laughton et al., 2003). Similar findings were observed with increased running speeds. Running at faster speed was found to significantly increase knee joint stiffness and leg stiffness, while the observed increases in ankle joint stiffness were considered less substantial (Arampatzis et al., 1999). This conflicting information regarding the interplay between foot strike pattern and leg stiffness make difficult to decide which is the most advantageous striking pattern from a running economy perspective.

Interestingly, most studies that directly investigated the effect of striking pattern on running economy propose that running economy is not affected by the striking pattern and its previously discussed effect on stiffness measures (Ardigò et al., 1995; Cunningham et al., 2010; Gruber et al., 2013; Perl et al., 2012). Gruber et al. (2013) proposed that adopting a rearfoot striking pattern might be advantageous for running at faster speeds according to their results. However, the observed percent differences favoring the rearfoot striking pattern were not considered adequate for inducing physiologically impactful running economy alterations for the average recreational runner. On the other hand, it was suggested that elite athletes may benefit from the potential positive effects of a rearfoot strike, since any running economy improvement may be of determining importance in terms of competition performance (Gruber et al., 2013). Similarly, in a modeling study, a rearfoot striking pattern was proposed as optimal in shod conditions in terms of energy conservation, while nonrearfoot striking patterns were preferable for equally sharing muscle work and minimizing local muscle fatigue. According to the authors of this study, rearfoot running was characterized as the most "versatile" 
running pattern and it was proposed that this versatility may explain its wide prevalence among runners of different levels and backgrounds (Miller and Hamill, 2015), since 75\% of runners make initial ground contact with their heel first (Gruber et al., 2013). Thus, although forefoot running appears to enhance the ability of the leg to store and reutilize elastic energy, the increased contractile costs due to increased muscle activation requirements, especially in the triceps surge muscle group, counterbalance the mechanical advantages and no differences can be observed between forefoot and rearfoot striking patterns (Perl et al., 2012).

\section{Footwear}

Another factor that appears to have an influence on the regulation of the various lower-limb stiffness measures are running shoes. Related studies have made comparisons between shod and barefoot running conditions (De Wit et al., 2000; Divert et al., 2005). The recent development of different types of minimalistic footwear as a means of simulating the barefoot running experience while providing protection from epidermal wounds also led to the performance of studies investigating the effect of running in minimalistic shoes on the regulation of lower-limb stiffness (Sinclair et al., 2016).

The specific interplay between joint stiffness and footwear condition has been investigated by only one study (Sinclair et al., 2016). In particular, Sinclair et al. (2016) reported that both at barefoot running and at running with minimalist-inspired footwear knee joint stiffness increased, while ankle joint stiffness decreased compared with conventional footwear. The observed increased knee joint stiffness at barefoot and barefoot-inspired footwear running was attributed to reduced total knee excursion as a consequence of the reduced stance time and the increased stride frequency. On the other hand, increased total ankle excursion was the cause of the reduced ankle joint stiffness (Sinclair et al., 2016).

The majority of the literature has been focused on the investigation of the effect of footwear condition on leg and vertical stiffness. The main finding of all the reviewed studies was that barefoot running or running at minimalist footwear is accompanied by increases in leg stiffness (De Wit et al., 2000; Divert et al., 2005; Divert et al., 2008; Lussiana et al., 2015; Shih et al., 2013; Sinclair et al., 2016). Given that leg stiffness is expressed by the ratio of Vertical Ground Reaction Forces to the magnitude of the leg compression expressed as the length deformation during stance (Lussiana et al., 2015), the recorded leg stiffness increases in barefoot and in minimally-shod running conditions can be attributed either to decreased leg compression through shorter ground contact times or to increased vertical ground reaction force values. The interplay between these two mechanisms presents variations with the final regulation of leg stiffness depending on the various possible combinations (De Wit et al., 2000; Divert et al., 2005; Divert et al., 2008; Hamill et al., 2011; Lussiana et al., 2015; Sinclair et al., 2013; Sinclair et al., 2016; Squadrone and Gallozzi, 2009). It appears that systematic exposure to barefoot running is correlated with reduced impact forces at initial contact with the ground, as part of a general movement optimization process (Crowell and Davis, 2011; Lussiana et al., 2015; Utz-Meagher et al., 2011). In this case, it is expected that the observed leg stiffness increases resulted mainly from reduced leg compression. For this reason, considering the subjects' training and preferred-footwear status may be helpful in any attempt of results' interpretation.

Another interesting perspective is the interplay between leg and vertical stiffness. In the studies of Shih et al. (2013) and Lussiana et al. (2015) it was reported that running barefoot or in minimal shoes was followed by an increase in leg stiffness, while the vertical stiffness did not present significant alterations (Lussiana et al., 2015; Shih et al., 2013). This type of locomotor response is part of the body's adaptation strategy to prevent any deviations from the habitual displacement of the COM (Ferris et al., 1999). Wearing or not wearing shoes and the type of footwear (i.e., minimalist vs. conventional) are stimuli that can alter the vertical displacement of COM (Ferris et al., 1999; Lussiana et al., 2015). Leg stiffness adjustments can compensate for the imposed perturbations from the footwear condition and, thus, the vertical displacement of the COM, expressed by vertical stiffness, remains unaltered (Ferris et al., 1999; Lussiana et al., 2015). Interestingly, this observation of increased leg stiffness and unaltered vertical stiffness was not verified by Divert et al. (2005) and Divert et al. (2008), who reported simultaneous increases in both vertical and leg stiffness during the barefoot and minimalist running conditions (Divert et al., 2005; Divert et al., 2008). It was proposed that the magnitude of the leg stiffness increase caused by barefoot running was not adequate for maintaining unaltered vertical stiffness. This increase in vertical stiffness was utilized as an argument favoring the superiority of barefoot running in terms of energetic cost (Divert et al., 2005). In the same direction, the increased total work production observed during running with thin diving socks, which simulates barefoot running imposed no metabolic penalty (Divert et al., 2008). This means that for a given amount of oxygen, more work can be produced during barefoot running. It was argued that from this perspective, barefoot running could 
be a more efficient type of locomotion (Divert et al., 2008). However, from the equations having been used by Divert et al. (2008) to calculate total work, it appears that $\mathrm{W}_{\mathrm{pot}}$, a component of $\mathrm{W}_{\text {tot }}$, is positively related with the vertical displacement of the COM. It might be that the COM at barefoot running should perform a greater vertical excursion compared to shod running and thus leading to increased total work production. This could be supported by arguments proposing that due to shoe sole geometry (thicker at the heel, thinner under the footballs), the foot's heel is elevated when wearing shoes compared to a more horizontal foot orientation relative to the ground at barefoot running (Lieberman et al., 2010). Additionally, a forefoot running pattern, which is usually adopted when running barefoot, also increases total work requirements due to increased joint excursions. The greater plantarflexion at the ankle joint before initial ground contact forces the footballs to touch the ground first. A dorsiflexion movement then follows allowing the heel to touch the ground, which is again followed by plantarflexion as the stance phase progresses (Utz-Meagher et al., 2011). At shod running however, this initial lowering movement is partially absent, due to the rearfoot striking prevalence (Utz-Meagher et al., 2011). The increased work requirements imposed by the different biomechanical characteristics between shod and barefoot running, although satisfied without metabolic penalty at barefoot running, do not exist during shod running. The potential disadvantage in terms of storage and return of elastic energy imposed by the shod condition compared to barefoot running is not translated into a $\mathrm{VO}_{2}$ increase, due to lower total work demands. Thus, although barefoot running is advantageous from the perspective of total work production for a given amount of consumed oxygen (net efficiency), it does not appear to be advantageous in terms of running economy, which is different from net efficiency, but it is a factor of major influence on running performance.

Moreover, barefoot running has been related with a factor of potential metabolic penalty, which is described by the "cost of cushioning” hypothesis (Franz et al., 2012). Particularly, it has been proposed that the materials and the shoe structure characteristics provide metabolic savings related with cushioning functions. In the case of barefoot running, these cushioning requirements should be reached by energy-consuming "active" processes (Franz et al., 2012; Tung et al., 2014).

Considering the above along with the recent development of light-weight materials, wearing light-weight running shoes that limit the disadvantage of added mass provides the energetically beneficial cushioning and at the same time imposes no additional total work production requirements. It could be said that technological evolutions in the field of shoe design and materials' development, particularly considering for the established relationship between running economy and stiffness, could counterbalance the previously mentioned reduced ability of storage and return of elastic energy at shod running compared to barefoot running.

\section{Neuromuscular training interventions}

Various neuromuscular training interventions have been related with increased musculotendinous stiffness. In particular, eccentric training was found to contribute to increases in passive Achilles tendon stiffness mainly due to intrinsic modifications of the musculotendinous tissue (Fouré et al., 2013). Isometric training-imposed alterations in the structure of the Achilles tendon, expressed by increased values of stiffness were considered responsible for performance improvements, as depicted by increased rate of torque development and decreases in electromyography delay of the vastus lateralis muscle (Kubo et al., 2001). After the completion of a plyometric training intervention, the decreases in the active series elastic component stiffness were linked with enhanced ability of the gastrocnemius musculotendinous unit to store and release elastic energy, which can justify the parallel jumping performance improvements (Fouré et al., 2011). Moreover, reported increases in passive gastrocnemius muscle stiffness not followed by increases in the Achilles tendon stiffness or any geometrical alterations provide evidence that plyometric training can induce changes in the mechanical characteristics of muscle tissue related with stretchshortening activities (Fouré et al., 2009). Similar results were also reported after a resistance training intervention focused on plantarflexion, which induced increase stiffness of the tendon structures (Kubo et al., 2002).

The discussed above studies, however, do not include measures or dependent variables directly related with running economy and subsequently with distance running performance. Interestingly, a study by Millet et al. (2002) reports correlations between hopping power and hopping stiffness as well as between hopping power and running economy after the completion of a combined endurance and strength training program. It was argued that the improvements in running economy relate with the behavior of stiffness. However, direct correlations between stiffness and running economy are not available in this study (Millet et al., 2002). More representative results are provided by Spurrs et al. (2003) who report that after a plyometric training program, simultaneous increases in running economy, lower-limb stiffness, jumping performance, and running time trial performance occurred, thus sup- 
porting the idea of neuromuscular training's positive effect on running economy through enhanced lower-limb stiffness and more efficient storage and return of elastic energy (Spurrs et al., 2003).

However, the potential of the various types of neuromuscular training to improve running economy and subsequently distance running performance by elevating lower-limb stiffness has not been extensively investigated. In most endurance running-related studies secondary variables, such as rate of force development and jumping performance, have been utilized as evidence that improvements in distance running performance can be correlated with enhanced lower-limb stiffness (Aagaard and Andersen, 2010). There are promising indications from the available data (Millet et al., 2002), but further research is required for firmer conclusions to be drawn.

\section{CONCLUSIONS}

Given the hypothesis that enhancing the lower limbs' ability for passive force generation would have a positive effect on running economy, it was the purpose of this comprehensive review to provide a list of modifiable factors with the potential to interact with this property. Lower-limb stiffness was one area of interest. Particularly, the interplay between the striking pattern, footwear conditions, and various neuromuscular training interventions and lower-limb stiffness was thoroughly discussed from the perspective of running economy. However, the multifactorial nature of running economy makes the establishment of firm conclusions rather difficult. Before proceeding to any interventions or modifications, coaches should take under consideration the anthropometric, physiological, and biomechanical profile of the runner and provide athlete-specific recommendations.

\section{CONFLICT OF INTEREST}

No potential conflict of interest relevant to this article was reported.

\section{REFERENCES}

Aagaard P, Andersen JL. Effects of strength training on endurance capacity in top-level endurance athletes. Scand J Med Sci Sports 2010;20 Suppl 2:39-47.

Arampatzis A, Brüggemann GP, Metzler V. The effect of speed on leg stiffness and joint kinetics in human running. J Biomech 1999;32:13491353.

Ardigò LP, Lafortuna C, Minetti AE, Mognoni P, Saibene F. Metabolic and mechanical aspects of foot landing type, forefoot and rearfoot strike, in human running. Acta Physiol Scand 1995;155:17-22.

Arellano CJ, Kram R. Partitioning the metabolic cost of human running: a task-by-task approach. Integr Comp Biol 2014;54:1084-1098.

Barnes KR, Kilding AE. Running economy: measurement, norms, and determining factors. Sports Med Open 2015;1:8.

Cavagna GA, Franzetti P, Heglund NC, Willems P. The determinants of the step frequency in running, trotting and hopping in man and other vertebrates. J Physiol 1988;399:81-92.

Conley DL, Krahenbuhl GS. Running economy and distance running performance of highly trained athletes. Med Sci Sports Exerc 1980;12: 357-360.

Crowell HP, Davis IS. Gait retraining to reduce lower extremity loading in runners. Clin Biomech (Bristol, Avon) 2011;26:78-83.

Cunningham CB, Schilling N, Anders C, Carrier DR. The influence of foot posture on the cost of transport in humans. J Exp Biol 2010;213:790-797.

Dalleau G, Belli A, Bourdin M, Lacour JR. The spring-mass model and the energy cost of treadmill running. Eur J Appl Physiol Occup Physiol 1998;77:257-263.

De Wit B, De Clercq D, Aerts P. Biomechanical analysis of the stance phase during barefoot and shod running. J Biomech 2000;33:269-278.

Divert C, Baur H, Mornieux G, Mayer F, Belli A. Stiffness adaptations in shod running. J Appl Biomech 2005;21:311-321.

Divert C, Mornieux G, Freychat P, Baly L, Mayer F, Belli A. Barefoot-shod running differences: shoe or mass effect? Int J Sports Med 2008;29:512518.

Farley CT, Morgenroth DC. Leg stiffness primarily depends on ankle stiffness during human hopping. J Biomech 1999;32:267-273.

Ferris DP, Liang K, Farley CT. Runners adjust leg stiffness for their first step on a new running surface. J Biomech 1999;32:787-794.

Fletcher JR, Esau SP, Macintosh BR. Economy of running: beyond the measurement of oxygen uptake. J Appl Physiol (1985) 2009;107:1918-1922.

Fouré A, Nordez A, Cornu C. Effects of eccentric training on mechanical properties of the plantar flexor muscle-tendon complex. J Appl Physiol (1985) 2013;114:523-537.

Fouré A, Nordez A, Guette M, Cornu C. Effects of plyometric training on passive stiffness of gastrocnemii and the musculo-articular complex of the ankle joint. Scand J Med Sci Sports 2009;19:811-818.

Fouré A, Nordez A, McNair P, Cornu C. Effects of plyometric training on both active and passive parts of the plantarflexors series elastic component stiffness of muscle-tendon complex. Eur J Appl Physiol 2011; 111:539-548.

Franz JR, Wierzbinski CM, Kram R. Metabolic cost of running barefoot versus shod: is lighter better? Med Sci Sports Exerc 2012;44:1519-1525.

Gruber AH, Umberger BR, Braun B, Hamill J. Economy and rate of carbo- 
hydrate oxidation during running with rearfoot and forefoot strike patterns. J Appl Physiol (1985) 2013;115:194-201.

Hamill J, Gruber AH, Derrick TR. Lower extremity joint stiffness characteristics during running with different footfall patterns. Eur J Sport Sci 2014;14:130-136

Hamill J, Moses M, Seay J. Lower extremity joint stiffness in runners with low back pain. Res Sports Med 2009;17:260-273.

Hamill J, Russell EM, Gruber AH, Miller R. Impact characteristics in shod and barefoot running. Footwear Sci 2011;3:33-40.

Kerdok AE, Biewener AA, McMahon TA, Weyand PG, Herr HM. Energetics and mechanics of human running on surfaces of different stiffnesses. J Appl Physiol (1985) 2002;92:469-478.

Kubo K, Kanehisa H, Fukunaga T. Effects of resistance and stretching training programmes on the viscoelastic properties of human tendon structures in vivo. J Physiol 2002;538(Pt 1):219-226.

Kubo K, Kanehisa H, Ito M, Fukunaga T. Effects of isometric training on the elasticity of human tendon structures in vivo. J Appl Physiol (1985) 2001;91:26-32.

Lacour JR, Bourdin M. Factors affecting the energy cost of level running at submaximal speed. Eur J Appl Physiol 2015;115:651-673.

Laughton CA, Davis IM, Hamill J. Effect of strike pattern and orthotic intervention on tibial shock during running. J Appl Biomech 2003;19: 153-168.

Lieberman DE, Venkadesan M, Werbel WA, Daoud AI, D'Andrea S, Davis IS, Mang'eni RO, Pitsiladis Y. Foot strike patterns and collision forces in habitually barefoot versus shod runners. Nature 2010;463: $531-535$

Lussiana T, Hébert-Losier K, Mourot L. Effect of minimal shoes and slope on vertical and leg stiffness during running. J Sport Health Sci 2015;4: 195-202.

Melcher DA, Paquette MR, Schilling BK, Bloomer RJ. Joint stiffness and running economy during imposed forefoot strike before and after a long run in rearfoot strike runners. J Sports Sci 2017;35:2297-2303.

Miller RH, Hamill J. Optimal footfall patterns for cost minimization in running. J Biomech 2015;48:2858-2864.

Millet GP, Jaouen B, Borrani F, Candau R. Effects of concurrent endurance and strength training on running economy and $\mathrm{VO}_{2}$ kinetics. Med Sci Sports Exerc 2002;34:1351-1359.

Moore IS. Is there an economical running technique? A review of modifiable biomechanical factors affecting running economy. Sports Med 2016;46:793-807.

Perl DP, Daoud AI, Lieberman DE. Effects of footwear and strike type on running economy. Med Sci Sports Exerc 2012;44:1335-1343.

Shih Y, Lin KL, Shiang TY. Is the foot striking pattern more important than barefoot or shod conditions in running? Gait Posture 2013;38:490494.

Sinclair J, Atkins S, Taylor PJ. The effects of barefoot and shod running on limb and joint stiffness characteristics in recreational runners. J Mot Behav 2016;48:79-85.

Sinclair J, Greenhalgh A, Brooks D, Edmundson CJ, Hobbs SJ. The influence of barefoot and barefoot-inspired footwear on the kinetics and kinematics of running in comparison to conventional running shoes. Footwear Sci 2013;5:45-53.

Spurrs RW, Murphy AJ, Watsford ML. The effect of plyometric training on distance running performance. Eur J Appl Physiol 2003;89:1-7.

Squadrone R, Gallozzi C. Biomechanical and physiological comparison of barefoot and two shod conditions in experienced barefoot runners. J Sports Med Phys Fitness 2009;49:6-13.

Tung KD, Franz JR, Kram R. A test of the metabolic cost of cushioning hypothesis during unshod and shod running. Med Sci Sports Exerc 2014;46:324-329.

Utz-Meagher C, Nulty J, Holt L. Comparative analysis of barefoot and shod running. Sport Sci Rev 2011;20:113-130. 